\title{
28 Geschlechtsspezifische Aspekte in der Psychotherapie
}

Martina Belz und Anita Riecher-Rössler

\section{Kernaussagen}

In der Psychotherapie ist die Berücksichtigung geschlechtsspezifischer Aspekte unabhängig von der psychotherapeutischen Orientierung eine wichtige Variable für das Gelingen des therapeutischen Prozesses und das Therapieergebnis. Dies betrifft Lebensbedingungen und soziale Realität, Gesundheit und Gesundheitsverhalten, die psychotherapeutische Behandlung selbst, Fragestellungen und Methoden der Psychotherapieforschung sowie die Inhalte und Strukturen der Aus- und Weiterbildung.

In der konkreten psychotherapeutischen Arbeit lassen sich fünf Ebenen beschreiben, die geschlechtsspezifisch Einfluß auf den Therapieprozeß und das Therapieergebnis haben können: 1. Menschenbild und therapeutische Orientierung; 2 interpersonelle Beziehungen und Kontextbedingungen; 3. Therapiebeziehung; 4. Therapietechniken und ziele und 5. Selbsterfahrung.

\section{Einleitung}

Psychische Gesundheit und Krankheit sowie deren Behandlung werden durch das Geschlecht maßgeblich beeinflußt. Auch in der Psychotherapie ist dabei sowohl die Berücksichtigung des „biologischen Geschlechts“ (englisch: „sex“), als auch des „sozialen Geschlechts" (englisch: „gender") bedeutsam.

Die Darstellung der Unterschiede zwischen den Geschlechtern kann zu einer ungewollten Dichotomisierung führen, indem Geschlechtsspezifisches hervorgehoben und dadurch verfestigt wird. Deshalb wird in Verbindung mit geschlechtsspezifischen Unterschieden immer wieder auf weitere Faktoren wie Familienstand, Bildungsstand oder lebensweltliche Rahmenbedingungen hingewiesen. Zum anderen werden, wo möglich, Unterschiede innerhalb der Geschlechtergruppen thematisiert, die genauso groß sein können wie zwischen den Geschlechtern. Auch auf die sich langsam entwickelnde Männergesundheitsforschung wird Bezug genommen, um neben frauenspezifischen auch die an die unterschiedlichen männlichen Lebensweisen gebundenen gesundheitlichen Risiken und Ressourcen offenzulegen (Kolip und Hurrelmann 2016; RKI 2014).

Trotz manchen Angleichungen in den letzten Jahren sind Frauen und Männer nach wie vor unterschiedlichen Lebensbedingungen ausgesetzt. Dabei lassen sich verschiedene Bereiche nennen, in denen es psychotherapierelevante geschlechtsspezifische Besonderheiten und Unterschiede zwischen Männern und Frauen gibt, die biologisch bedingt und/oder sozial wie auch psychologisch geprägt sind. Unabhängig von der Therapieschulzugehörigkeit des Therapierenden und der Störung des Patienten bzw. der Patientin sollten Psychotherapeutinnen und Psychotherapeuten die entsprechenden theoretischen und empirischen Erkenntnisse rezipieren, fortlaufend aktualisieren und in das psychotherapeutische Arbeiten integrieren. Das Wissen über psychotherapierelevante Geschlechtsunterschiede läßt sich in folgende sieben Punkte unterteilen:

1. Lebensbedingungen und soziale Realität

2. Gesundheitszustand, Gesundheitsverständnis und Gesundheitsverhalten 
3. Häufigkeit und Verbreitung von psychischen Störungen

4. Entwicklung und Verlauf von psychischen Störungen

5. Psychotherapeutische Versorgung und Behandlung

6. Psychotherapieforschung

7. Aus- und Weiterbildung

\section{Lebensbedingungen und soziale Realität}

\section{Einflußfaktor Berufstätigkeit}

Zunehmend mehr Frauen nehmen am Erwerbsleben teil, die Erwerbsmuster von Männern und Frauen unterscheiden sich aber immer noch erheblich. Am ausgeprägtesten ist dies in der Gruppe der Verheirateten. Nach der Eheschließung kommt es häufig zu einer Traditionalisierung der Arbeitsteilung, wobei heute an die Stelle des Modells der ErnährerHausfrauen-Familie das Vollzeit-Teilzeit-Modell getreten ist. Während Männer nach der Geburt eines Kindes weiterhin erwerbstätig bleiben und sich so berufliche Aufstiegschancen und die Möglichkeit zur Weiterentwicklung sichern, sind Frauen häufiger in Berufen mit niedrigerem Status, als Teilzeitbeschäftigte und in hierarchisch untergeordneten Positionen zu finden. Bei Frauen mit Migrationshintergrund sind diese traditionellen Erwerbsmuster noch häufiger verbreitet.

Das Einkommen von Frauen liegt in Europa immer noch deutlich unter dem von Männern. In Führungspositionen sind Frauen nach wie vor extrem unterrepräsentiert - all dies, obwohl inzwischen weltweit Frauen den Männern in Sachen Bildung den Rang ablaufen.

Die sich in diesen Daten widerspiegelnde geschlechtsspezifische Segmentierung der Arbeitswelt ist in den Ländern am wenigsten ausgeprägt, in denen es ein gut ausgebautes System der Kinderbetreuung gibt und in denen gesetzliche wie auch steuerliche Regelungen die Berufstätigkeit von Frauen vor allem mit Kindern fördern. Aber es ist nicht nur die Betreuung eigener Kinder, die dazu führt, daß Frauen in der Erwerbstätigkeit weniger Fuß fassen als Männer. Ergebnisse der EUROFAMCARE-Studie zeigen, daß international der Frauenanteil bei den pflegenden Angehörigen bei 68\% liegt (Kofahl, Mestheneo Triantafillou 2005). Frauen erleben die mit der Pflege von Angehörigen verbundenen Belastungen anders als Männer: Männer sehen darin eher eine Managementaufgabe (Langehennig et al. 2012), grenzen sich klarer ab und nehmen viel früher und deutlich mehr professionelle Unterstützung und Hilfe in Anspruch als pflegende Frauen.

Insgesamt leisten Männer wesentlich häufiger bezahlte und Frauen entsprechend mehr unbezahlte Arbeit. Die Kehrseite ist, daß Männer dadurch meist weniger in familiäre Beziehungen - auch mit ihren Kindern - integriert sind und weniger legitimierte Möglichkeiten haben, berufliche Unterbrechungen zur Neuorientierung zu nutzen.

Die Mehrfachbelastungen, denen Frauen ausgesetzt sind, führen genauso wie der auf Männern lastende Karrieredruck bei beiden Geschlechtern immer wieder zu Überforderungssyndromen, die psychotherapeutische Unterstützung notwendig machen. Auch kann die (finanzielle) Abhängigkeit von Frauen mit Kindern deren Autonomie und Selbstwert beeinträchtigen und so psychische Störungen fördern. Diese sozialen Rahmenbedingungen, aber auch der Umgang mit den damit zusammenhängenden Belastungen können die Entscheidung zur Aufnahme einer Psychotherapie in verschiedener Weise beeinflussen. Eine Vollzeitberufstätigkeit kann aufgrund der zeitlichen Einschränkungen eine Psychotherapie ebenso erschweren wie das Angebundensein durch Kinder oder pflegebedürftige Angehörige. Knappe finanzielle Mittel machen Psychotherapie in Ländern, in denen sie keine Regelleistung der Versicherungen darstellt, für Menschen mit niedrigem Einkommen - und das sind immer noch häufiger Frauen - kaum erschwinglich. Das männliche Rollenstereotyp, das Stärke, Autonomie und Kontrolle fordert, ist mit der 
Aufnahme einer Psychotherapie und der Vorstellung von Schwäche und Unterstützungsbedarf z.T. schwer vereinbar.

\section{Einflußfaktor Gewalt}

Gewalt gilt als eines der bedeutsamsten Gesundheitsrisiken, mit denen Frauen und Mädchen auf der ganzen Welt konfrontiert sind (Garcia-Moreno \& Riecher-Rössler 2013). In Europa geht es vor allem um häusliche und sexualisierte Gewalt. Diese geht zum Grossteil von Männern aus, mit denen die Frauen bekannt, befreundet oder verheiratet sind.

Auch bezogen auf die Angst, zum Opfer von Gewalt zu werden, sind geschlechtsspezifische Unterschiede feststellbar. Vor dem öffentlichen Raum als tendenziell gefährlich werden Mädchen eher gewarnt, Jungen eher dazu aufgefordert, ihn zu nutzen und sich möglichen Gefahren zu stellen (Schwind et al. 2001). Besonders häufig erleben Migrantinnen und Flüchtlingsfrauen Gewalt (Khelaifat und Schröttle 2008).

\section{Einflußfaktor Alter und Lebensform}

Geschlechtsspezifisch relevant sind auch die Verschiebung der Altersstruktur in der Bevölkerung, von der Frauen wegen der höheren Lebenserwartung stärker betroffen sind, und die damit einhergehenden psychischen Erkrankungen. Der Wandel der Lebensformen (weniger Kinder, mehr Singles, mehr Alleinerziehende) wirkt sich geschlechtsspezifisch unterschiedlich aus (z.B. Überforderungssyndrome und zunehmendes Armutsrisiko bei Frauen) und beeinflußt die in einer Psychotherapie relevanten Themen.

\section{Gesundheitszustand, Gesundheitsverständnis und Gesundheitsverhalten}

Was Männer und Frauen unter Gesundheit verstehen, bestimmt auch, wann sie sich als krank und behandlungsbedürftig wahrnehmen, was sie tun, um ihre Gesundheit zu erhalten bzw. wiederherzustellen. Ein Blick auf geschlechtsspezifisches Inanspruchnahmeverhalten bei körperlichen Erkrankungen gibt zudem Hinweise auf Aspekte, die auch bei der Versorgung von Männern und Frauen mit psychischen Problemen eine Rolle spielen könnten.

Die höhere Mortalität der männlichen Bevölkerung vor dem 65. Lebensjahr wird vor allem als Folge ihres spezifischen geschlechtsstereotypen Risikoverhaltens (z.B. risikoreiche Sportarten, Arbeitsunfälle, Suchtmittelmissbrauch) erklärt. Hinz (2007) weist darauf hin, daß einige der beschriebenen gesundheitsriskanten Verhaltensweisen (z.B. riskant und schnell Auto fahren oder waghalsig Sport treiben, um Grenzen auszutesten und sich als männlich stark und furchtlos zu zeigen) als Versuch verstanden werden können jugendspezifische Entwicklungsaufgaben zu meistern. Geschlechtsunterschiede zeigen sich auch im sexuellen Risiko- und Schutzverhalten. Männer können ohne das Einverständnis von Frauen Kondome benutzen, umgekehrt ist das nicht möglich. Am letztgenannten Beispiel wird deutlich, daß das gesundheitliche Risiko- und Schutzverhalten auch durch geschlechtsspezifische Abhängigkeiten beeinflußt sein kann.Männer bewerten ihren Gesundheitszustand im Durchschnitt besser und sind mit ihrer Gesundheit zufriedener als Frauen, wobei Alter, Höhe des Einkommens, beruflicher Bildungsabschluss und Erwerbstätigkeitsstatus noch stärker mit dem Urteil korrelieren als das Geschlecht. Auch beim Inanspruchnahmeverhalten zeigen sich relevante Geschlechtsunterschiede. Bereits im Alter von 15 Jahren suchen Mädchen und Frauen öfter ärztliche Hilfe auf und werden mehr krankgeschrieben als Jungen und Männer. Sie registrieren mehr Symptome und zeigen eine aktivere Beschwerdeschilderung. Frauen nehmen die Dienstleistungen von Krankenhäusern deutlich häufiger in Anspruch als Männer. Dies ist auch dann noch der Fall, wenn die Zahlen alterskorrigiert sind und Krankenhausaufenthalte wegen Entbindung unberücksichtigt bleiben. Männer verbringen jedoch durchschnittlich mehr Tage im Krankenhaus. Wenn 
Männer zum Arzt gehen, werden sie in Bezug auf ihre Beschwerden ernster genommen und gründlicher untersucht. Frauen bekommen hingegen häufiger und mehr Medikamente verschrieben (Stürzer und Cornelißen 2005). Unterschiede zwischen den Geschlechtern bestehen weiterhin in der Art der Medikamenteneinnahme: Während Neuroenhancement zur Leistungssteigerung vor allem Männer anspricht, werden Medikamente zur Verbesserung der Stimmung oder zum Abbau von Ängsten und Nervosität häufiger von Frauen genutzt (IGES Institut, 2015), wohl auch den häufigeren depressiven und Angsterkrankungen bei Frauen entsprechend (s.u.).

Männer haben ein funktionaleres Gesundheitsverständnis, d.h. Gesundheit bedeutet für sie in erster Linie Leistungsfähigkeit, körperliche Funktionsfähigkeit und „Abwesenheit von Krankheit" (Bengel und Belz-Merk 1997). Sie rauchen mehr, konsumieren mehr Alkohol sowie illegale Drogen und sind deutlich häufiger übergewichtig als Frauen. Diese achten zwar mehr auf die Ernährung, teilweise aber im Sinne einer Fehlernährung durch zu wenig Nahrungszufuhr. Sie treiben weniger Sport als Männer. Frauen haben eher ein ganzheitliches, positives, an Wohlbefinden orientiertes Gesundheitsverständnis. Sie nehmen ihre Krankheitssymptome im Allgemeinen besser wahr als Männer und haben auch eine bessere Krankheitsbewältigung. Frauen wenden sich eher an Hausärzte, während Männer verstärkt Fachärzte konsultieren (WHO-Studie „Women's Mental Health“ 2000). Frauen nehmen auch häufiger "Laienhilfen" wie Selbsthilfegruppen und -literatur in Anspruch, sie besuchen Esoterikmessen und kaufen eher in Bioläden ein. Weitere Erklärungsansätze für die berichteten Geschlechtsunterschiede im Gesundheitszustand und -verhalten liegen in den spezifischen Erfahrungen, die Männer und Frauen innerhalb des Gesundheitssystems machen, aber auch in methodischen Artefakten bei Erhebungsverfahren.

\section{Häufigkeit und Verbreitung psychischer Störungen}

Nach den Daten einer aktuellen repräsentativen Bevölkerungsstichprobe (Wittchen et al 2012), die mit international anerkannten und zuverlässigen Untersuchungsmethoden auf der Grundlage aktueller Klassifikationssysteme erhoben wurden, erfüllten im Jahre 2011 in einem Zeitraum von 12 Monaten 33,3\% der deutschen Bevölkerung die Kriterien für klinisch bedeutsame psychische Störungen nach DSM-IVTR. Aufgeteilt nach Geschlechtern betraf dies Frauen zu 35,9\% und Männer zu 30,7\%. Was die Gesamthäufigkeit psychischer Störungen angeht, zeigte sich damit kein gravierender Geschlechtsunterschied, wohl aber bezüglich der Diagnosespektren.

Daß es bei vielen psychischen Erkrankungen, die beide Geschlechter betreffen, große Häufigkeitsunterschiede gibt, ist schon länger bekannt (Übersicht in Riecher-Rössler und Bitzer 2005). So leiden Frauen deutlich häufiger an Eßstörungen (9 F:1 M) wie Anorexie und Bulimie. Depressionen sind bei Frauen doppelt so häufig wie bei Männern, und zwar von den leichteren depressiven Zuständen bis hin zu den schweren unipolaren affektiven Erkrankungen. Auch in neueren Studien zeigen sich ähnliche Geschlechtereffekte und ein deutlicher Zusammenhang mit dem Sozialstatus. Auch viele Arten von Angststörungen (einschließlich Agoraphobie, Panikerkrankung und soziale Phobie) sind bei Frauen häufiger. Ebenso werden Somatisierungsstörungen, Borderline-Persönlichkeitsstörungen (3 F:1 M) und dissoziative Identitätsstörungen (3-9 F:1 M) bei ihnen häufiger diagnostiziert. Nach einem Trauma entwickeln Frauen eher eine posttraumatische Belastungsstörung. Von einer Medikamentenabhängigkeit sind Frauen dreimal häufiger betroffen als Männer. Insgesamt nehmen sie doppelt so häufig wie Männer psychoaktive Medikamente ein, was z.T. durch die häufigere Depression (s.u.) und deren psychopharmakologische Behandlung zu erklären ist. Da aber Medikamentenabhängigkeit meist im Rahmen einer ärztlichen Behandlung beginnt und problematische Dauerverschreibungen von Benzodiazepinen weitgehend ( $\mathrm{zu}$ über $80 \%$ ) konsensuell zwischen Patient bzw. Patientin und einem einzigen Arzt erfolgen, 
kann dies als Hinweis für ein geschlechtsspezifisch unterschiedliches

Verschreibungsverhalten von Ärzten und Ärztinnen verstanden werden (Holzbach et al. 2010).

Bei Männern häufiger sind die meisten Persönlichkeitsstörungen, insbesondere antisozialer Art (Übersichten in Riecher-Rössler und Bitzer 2005) sowie der Großteil an Paraphilien. Erhöhte Prävalenzzahlen in den Persönlichkeitsstörungen bei Frauen finden sich bei der dependenten und der histrionischen Persönlichkeitsstörung. Lediglich bei bipolaren Erkrankungen und schizophrenen Störungen sind Männer wie Frauen nahezu gleich häufig betroffen. Gerade die geschlechtsspezfische Dominanz bestimmter Störungsbilder und die Tatsache, daß Frauen in den weniger biologisch bestimmten Störungen überwiegen, macht den Einfluß psychosozialer Faktoren auch bei psychischen Störungen plausibel.

Frauen verüben auch häufiger Suizidversuche als Männer. Bei Männern dagegen sind vollendete Suizide häufiger, ebenso wie Abhängigkeiten von Substanzen außer Medikamenten - 70\% der Alkoholabhängigen sind Männer. Die signifikant höhere Suizidrate insbesondere bei jungen und älteren Männern wird von manchen Autoren zusammen mit der hohen Alkoholismusrate als Äquivalent der weiblichen Depression angesehen, da Männer im Falle einer Depression entsprechend der männlichen Geschlechtsrollenerwartung im Vergleich zu Frauen deutlich seltener professionelle Hilfe in Anspruch nehmen und stattdessen Alkohol und als letzten Ausweg den Suizid als Bewältigungsstrategie einsetzten (Möller-Leimkühler 2000, Wolfersdorf und SchulteWefers 2005).

Gutiérrez-Lobos et al. (2000) fanden in einer Studie zum sogenannten „gender gap in depression" die höchste Depressionsrate bei nichtberufstätigen geschiedenen Frauen, die niedrigste bei berufstätigen verheirateten Männern.

Meshkat et al. (2010) diskutieren, daß es aufgrund der Diagnosekriterien in den gängigen Diagnosemanualen zu einer Unterschätzung der Depressionsraten bei Männern kommen könnte. Spezifisch männliche Symptome wie Aggressivität, Verärgerung, Irritabilität, Feindseligkeit oder erhöhtes Risiko- und Suchtverhalten könnten die gängigen Depressionssymptome überdecken. Das klinische Bild einer Depression weist demnach für Männer und Frauen spezifische Symptome auf, die bei einer Diagnosestellung berücksichtigt werden müßten. Möglicherweise gilt dies auch für andere Störungsbilder.

Zudem ist kritisch zu diskutieren, daß für die Diagnose einer psychischen Störung neben standardisierten, von Experten bzw. Expertinnen entwickelten Diagnosesystemen auch die Normalitätsbilder einer Gesellschaft relevant sind. Diese legen Frauen und Männer auf "gute \& richtige“ Eigenschaften fest. Eindrücklich ist die Bedeutung dieser Normalitätsbilder in der klassischen Fragebogenstudie von Broverman et al. (1970) an drei Gruppen von Psychotherapeutinnen und Psychotherapeuten gezeigt worden. Diese hatten die Aufgabe anzugeben, welche Merkmale sie mit dem Bild eines gesunden Mannes (Gruppe 1), eines gesunden Menschen (Gruppe 2) und einer gesunden Frau (Gruppe 3) verbinden. Danach entspricht das Bild eines gesunden Mannes mit den Merkmalen Unabhängigkeit, Rationalität und Gelassenheit dem Bild eines gesunden Menschen (Gruppe 2). Das Bild einer gesunden Frau (Gruppe 3) wurde mit den Eigenschaften „gefühlvoll, nachgiebig und wenig aggressiv" beschrieben und entsprach genau dem Bild eines psychisch gestörten Menschen. Die Studie wurde in ähnlicher Form repliziert (Nesbitt und Penn 2000) und macht unmittelbar einsichtig, wie gesellschaftliche Normalitätsbilder, auch Einfluss auf das Diagnostizieren und Pathologisieren von Therapeuten und Therapeutinnen haben können. Wenn Frauen mit Eigenschaften wie Passivität, Sensibilität, Emotionalität, Kooperationswilligkeit, Fürsorglichkeit und Zuständigkeit für Beziehungspflege ausgestattet werden und Männern Merkmale wie Aggressivität, Sachorientierung, Konfliktfreudigkeit, Autonomie, Aktivität und Rationalität zugeschrieben werden, dürfte diese Haltung auch 
Therapieziele und -ergebnisse beeinflussen. Trotz Enttraditionalisierung sind diese Geschlechtsrollenstereotypien über Zeit und Kulturen hinweg erstaunlich stabil (Glick et al. 2004).

Weder Frauen noch Männer sind also insgesamt das kränkere Geschlecht, sie weisen lediglich unterschiedliche Krankheitsprofile auf, in die geschlechtsrollenstereotypische Aspekte einfließen.Frauen geben aber insgesamt öfter als Männer an, wegen psychischer Probleme und Störungen in Behandlung gewesen zu sein (21 vs. $10 \%$ ) oder sich vorstellen zu können, dies im Bedarfsfall zu tun (62 vs. 44\%). Unter den Psychotherapiepatienten haben Männer nur einen Anteil von etwa 30\% (Dorrmann et al. 2014). Trotz gesellschaftlicher Veränderungen sind immer noch geschlechtsrollenspezifische Vorstellungen verbreitet, die es Männern erschweren, Psychotherapie in Anspruch zu nehmen, da dies gleichbedeutend mit dem Zeigen von Schwäche ist. Das Offenlegen von Problemen, die eigene Verletzlichkeit zeigen, Gefühle ausdrücken und sich einem Therapeuten oder einer Therapeutin anvertrauen, entsprechen mehr dem weiblichen Problemlöseverhalten (Mahalik et al. 2003). Dies gilt noch mehr für paar- und/oder familientherapeutische Settings (Owen et al. 2009).

36\% aller Frühberentungen bei Frauen erfolgen aufgrund psychischer Erkrankungen (bei den Männern sind es 25\%). Insbesondere Frauen sind von Arbeitsunfähigkeit aufgrund von psychischen Störungen betroffen. Laut DAK Gesundheitsreport von 2015 liegt der Anteil der Frauen mit einer Krankschreibung aufgrund einer psychischen Störung bei 19,9\% verglichen mit 13,4\% bei den Männern (IGES Institut 2015). Möglicherweise sind diese Unterschiede durch die unterschiedliche Verteilung von Diagnosen mit mehr depressiven und Angsterkrankungen bei Frauen und/oder durch das unterschiedliche Inanspruchnahmeverhalten bedingt.

\section{Entwicklung und Verlauf psychischer Störungen}

Im Kindes- und Jugendalter werden bei Mädchen häufiger internalisierende Störungen diagnostiziert, bei Jungen eher externalisierende Verhaltensweisen. Nach Einsetzen der Pubertät wird der Anteil von Mädchen mit Depression immer größer, Jungen dominieren dagegen bei Lern- und Leistungsstörungen, AufmerksamkeitsdefizitHyperaktivitätsstörungen sowie Delinquenz und Störungen durch Substanzgebrauch (Steinhausen 2010; Fuchs et al. (2013).

Warum Frauen etwa doppelt so häufig an Depressionen leiden wie Männer ist nach wie vor nicht ganz geklärt. Verschiedene Theorien (Übersicht bei Riecher-Rössler 2013, 2015) gehen davon aus, daß die geschlechtsspezifische Sozialisation von Knaben und Mädchen das spätere geschlechtsspezifische Rollenverhalten prägt. Riecher-Rössler argumentiert, Mädchen würden eher zu Passivität, erlernter Hilflosigkeit und geringerem Selbstvertrauen erzogen, Knaben dagegen eher zu aktivem Coping. In der Folge tendierten Frauen deshalb dazu, Konflikte eher zu internalisieren und mit Grübeln, Schuldgefühlen und Depressionen zu reagieren, während Männer eher externalisierten und entweder aktive oder gar aggressive Bewältigungsstrategien suchten oder zu Alkohol griffen (Belz und RiecherRössler 2008, Riecher-Rössler et al., 2003, 2015). Das Depressionsrisiko bei Frauen werde aber auch erhöht durch ihren geringeren sozialen Status, ihre stärkeren Abhängigkeiten, das Erleben von häuslicher Gewalt und sexuellem Mißbrauch, das Erleben von Armut (insbesondere als alleinerziehende Mutter) etc.

Wichtig ist in diesem Zusammenhang auch eine große Studie der Weltgesundheitsorganisation (WHO), die Women's Mental Health Survey (Seedat et al. 2009), die in 15 Ländern auf den verschiedensten Kontinenten dieser Erde durchgeführt wurde. Depressive Erkrankungen waren bei Frauen überall etwa doppelt so häufig wie bei Männern. In einigen wenigen Ländern war dieser Geschlechtsunterschied bei den jüngeren 
Jahrgängen jedoch geringer ausgeprägt als bei den älteren - und zwar interessanterweise in denjenigen Ländern, wo sich in den letzten Jahren die Geschlechterrollen von Frauen und Männern angenähert hatten.

Ein weiterer wichtiger Risikofaktor für die Entwicklung einer psychischen Störung und Anlaß, sich in psychotherapeutische Behandlung zu begeben, ist das häufig berichtete geringere Selbstwertgefüh/ von Frauen. In verschiedenen Studien zeigten sich Frauen stärker von positivem Feedback abhängig als Männer, sie wiesen eine geringere Handlungsorientiertheit auf und ein geringeres Durchsetzungsvermögen. Auf der anderen Seite zeigten Frauen offensichtlich stärkere Extraversion, Hilfsbereitschaft, Empathiefähigkeit und Beziehungsorientierung. All dies können prinzipiell Stärken von Frauen sein, die aber zu stärkeren Belastungen aus dem sozialen Netz führen können (Übersicht bei Belz und Riecher-Rössler 2008, Riecher-Rössler 2006, 2015).

Frauen unterscheiden sich im Mittel von Männern auch bezüglich ihres emotionalen Ausdrucks. So sprechen sie eher über ihre Gefühle, drücken diese im Allgemeinen auch intensiver aus und erinnern eher gefühlsbetonte Situationen. Frauen werden aber auch von negativen Gefühlen anderer stärker berührt, was sie vulnerabler macht. Sie zeigen häufiger und stabiler Angst, Niedergeschlagenheit und Trauer und neigen eher zu Besorgnis. Dagegen können sie weniger gut direkte - zum Teil aber mehr verbale - Aggressivität zeigen. Sie schämen sich häufiger und intensiver, insbesondere bezüglich ihres Körpers, ihrer sozialen Kompetenz und ihrer Leistungen. Und vor allem fühlen sie sich häufiger schuldig (Übersicht in Belz und Riecher-Rössler 2008, Riecher-Rössler 2003, 2013).

Bis dato gibt es keine hinreichende Erklärung, ob diese geschlechtsspezifischen Unterschiede eher genetisch/familiäre, biologische oder psychosoziale Hintergründe haben. Eine Perspektive, die all diese Faktoren in den Blick nimmt, erscheint der Komplexität am angemessensten.

\section{Psychotherapeutische Versorgung und Behandlung}

Erkenntnisse über die Bedeutung der Kategorie Geschlecht, die in die konkrete Arbeit mit Patienten und Patientinnen einzubeziehen sind, lassen sich auf fünf Ebenen beschreiben und zusammenfassen:

1. Ebene des Menschenbilds und der therapeutischen Orientierung: Wie beschreiben und erklären verschiedene Therapieschulen die Unterschiede der Geschlechter in den theoretischen Konzepten zum psychischen Funktionieren und zur geschlechtsspezifischen Entwicklung, zu Auftretenshäufigkeit und Erscheinungsbild psychischer Störungen?

2. Ebene der interpersonellen Beziehungen und der Kontextbedingungen des Patienten bzw. der Patientin: Wie gehen weibliche und männliche Bezugspersonen des Patienten bzw. der Patientin mit der Störung um?

3. Ebene der Therapiebeziehung: Wie beeinflussen geschlechtsspezifische Unterschiede in den Erwartungen von Therapeuten und Therapeutinnen hinsichtlich Partnerschaft, Arbeitsfähigkeit etc. gegenüber Patientinnen und Patienten die Therapiebeziehung?

4. Ebene der Therapietechniken und -ziele: Zeigen bestimmte therapeutische Techniken, aber auch Psychotherapieverfahren eine geschlechtsspezifische Wirksamkeit? Werden bestimmte Techniken in Abhängigkeit vom Geschlecht häufiger eingesetzt? Gibt es geschlechtsspezifisch zuordnungsfähige thematische oder technische Sperren? Lassen sich sinnvolle geschlechtsspezifische Therapieziele formulieren?

5. Ebene der Selbsterfahrung: Was traue ich mir als Therapeut bzw. Therapeutin zu und was nicht? Wo bin ich besonders empfindsam oder verführbar, und was hat das mit meiner geschlechtsspezifischen Sozialisation zu tun? 
Die genannten Aspekte werden im Folgenden unter Berücksichtigung schulenspezifischer Perspektiven integrativ und störungsübergreifend dargestellt.

\section{Menschenbild und therapeutische Orientierung}

Was haben die traditionellen psychotherapeutischen Schulen zur Aufklärung der Geschlechtsunterschiede beigetragen? Die klassische Psychoanalyse hat als Erklärungsansatz für die psychologischen Geschlechtsunterschiede "in ihren Anfängen als wohl bekanntestes Konzept das des Penisneids vorgelegt. Dieses postuliert, stark vereinfacht, daß aus der frühen Erfahrung dieses mangelnden Körperglieds das mangelnde Selbstwertgefühl der Frau und ihre Neigung zu bestimmten psychischen Erkrankungen entstehe - ein Konzept, das durch keinerlei Empirie belegt wurde und das doch die Basis dafür gelegt hat, daß sich Generationen von Patientinnen - und auch Therapeutinnen - mit ihrem vorgeblichen Mangel abfinden mußten. Denn ohne Überwindung des Penisneids mit Akzeptanz der passiven Objektrolle des Weibes war die psychische Gesundheit einer Frau gefährdet - so die psychoanalytische Theorie. Frauen, die die passive Objektrolle nicht akzeptierten, drohten an einem Männlichkeitskomplex mit Frigidität oder an Neurose zu erkranken. Auch die häufigere Depression der Frauen wurde zum Teil durch die narzißtische Kränkung aufgrund des fehlenden Penis erklärt (Freud 1925)" (zitiert nach Riecher-Rössler 2003).

Modernere Konzepte wurden von feministischen Psychoanalytikerinnen vorgelegt (vergleiche z.B. Riecher-Rössler 2003). So wagt zwar Karin Horney (1973) nicht, die Existenz eines Penisneids zu hinterfragen, sie unterscheidet aber zumindest zwischen einem „primären“ Penisneid aufgrund des biologischen Mangels und einem „sekundären“ Penisneid, mit dem Frauen auf ihre faktischen sozialen Beschränkungen reagierten. Sehr viel weiter geht Chodorow (1978). Sie geht davon aus, daß das so genannte asymmetrische Parenting in unserer Gesellschaft mit eine Rolle für die Entstehung psychischer Störungen spielt. Damit meint sie die Tatsache, daß in unserer Gesellschaft Kinder vor allem von den Müttern, also Frauen, erzogen würden und dies dazu führe, daß die Identitätsbildung bei Knaben und Mädchen unterschiedlich erfolge. Knaben müssten sich eher vom Weiblichen abgrenzen und lernten dadurch Autonomie, während Mädchen diese Notwendigkeit nie in gleichem Maße spürten und dadurch eher in symbiotischer Abhängigkeit verharrten. Hinzu komme, daß sie sich mit der Mutter als Frau identifizierten und Frauen in unserer Gesellschaft als minderwertig betrachtet würden.

In der Verhaltenstherapie ist die Geschlechterfrage erst in den letzten Jahren theoretisch diskutiert worden. Dabei wurde deutlich, daß es nicht ausreicht, eine geschlechterneutrale Theorie zu vertreten wie es die Verhaltenstherapie lange Zeit für sich in Anspruch genommen hat. In Deutschland besteht seit 1986 in der Deutschen Gesellschaft für Verhaltenstherapie die Arbeitsgemeinschaft "Frauen in der psychosozialen Versorgung“. Die AG macht auf die besondere Situation von Frauen im psychosozialen Bereich aufmerksam und setzt sich für eine Gleichstellung in Forschung, Theorie und psychosozialer Praxis ein. Ein Arbeitsschwerpunkt ist das Thema „Sexuelle Übergriffe in Therapie und Beratung".

Kognitive Theoretiker haben sich mit dem Einfluß des Geschlechts auf Kognitionen, Verhalten und Emotionen beschäftigt. Sie sehen eine der Ursachen für die bei Frauen häufiger beobachtete Passivität und gelernte Hilflosigkeit in der geschlechtsrollenspezifischen Sozialisation. Auch betonen sie, daß Frauen Ereignisse sowie die möglichen Bewältigungsmöglichkeiten anders bewerten als Männer und aufgrund dieser anderen nämlich negativeren - Bewertung eher Depressionen entwickeln. Sie beschreiben den so genannten negativen kognitiven Stil von Frauen, in dessen Rahmen Frauen für negative Ereignisse eher stabile, globale und vor allem internale Kausalattributionen entwickeln. Das heißt, Frauen glauben häufiger als Männer, daß sie für ein negatives Ereignis verantwortlich 
seien und daß das immer und in allen Bereichen so sei. Dies - so die kognitiven Theorien führe auch dazu, daß Frauen weniger glaubten, etwas durch aktive Beeinflussung ändern zu können, deswegen auch weniger motiviert für solche Änderungen seien, was den Verlauf der Depression negativ beeinflusse (Übersicht in Riecher-Rössler 2005).

Auch an diesen kognitiven Theorien wurde zum Teil Kritik geübt, insbesondere dahingehend, daß die tatsächlichen Lebensbedingungen von Frauen in ihrer Schwierigkeit ebenso häufig unterschätzt werden wie der soziale Kontext, in dem die Neigung zu dysfunktionalen Kognitionen entstehe.

Die systemische Familientherapie hat sich eher wenig mit dem Einfluß von Geschlechterrollen auseinandergesetzt (vergleiche dazu Belz und Riecher-Rössler 2008), obwohl Geschlechterrollen gerade für die Struktur und Funktionsweise von Familien besonders wichtig sind. Wie Willutzki (2001) betont, wird damit auch die Machtasymmetrie in Familien in der systemischen Therapie nicht thematisiert. Konzepte wie „Komplementarität“, „Zirkularität" oder „Neutralität“ und „Allparteilichkeit" trivialisierten die Unterschiede zwischen Verhaltensweisen und ihren Konsequenzen. „Geht man etwa davon aus, daß nicht zu entscheiden ist, ob sie nörgelt, weil er trinkt, oder aber er trinkt, weil sie nörgelt, so erscheinen beide Reaktionsweisen als gleichwertig in ihren Folgen. Im Extremfall gilt dies auch für Gewaltbeziehungen: Ihr Widerspruch und Nörgeln scheint seinen Schlägen gleichwertig" (Willutzki 2001: 703).

Sowohl systemische als auch psychoanalytische und tiefenpsychologische Schulen tendieren darüber hinaus dazu, die Ursachen von Problemen und psychischen Störungen in der Kindheit zu lokalisieren und implizit oder explizit Mütter hauptverantwortlich zu machen.

Die humanistischen/experientiellen Therapieverfahren richten sich mit ihren anthropologischen Grundannahmen am Individuum aus. Eine Auseinandersetzung mit geschlechtsspezifischen Fragestellungen fehlt dementsprechend bzw. wurde gar nicht als notwendig erachtet.

Insgesamt haben sich die großen psychotherapeutischen Schulen zwar zum Teil mit der Erklärung der Geschlechtsunterschiede bei psychischen Erkrankungen beschäftigt und zum Teil auch versucht, dies in ihrer Konzeptbildung zu berücksichtigen. Viele dieser Konzepte wie etwa die frühen psychoanalytischen - sind aber deutlich durch den jeweiligen Zeitgeist geprägt und müssen aus heutiger Sicht kritisch hinterfragt werden. Auch die geschlechtsneutralen individuumszentrierten Ansötze der humanistisch-erlebnisorientieren Verfahren und modernere Konzepte lassen eine kritische Auseinandersetzung mit gesellschaftlichen und kulturellen Einflüssen sowie sozialen Realitäten weitgehend vermissen oder werden wie bei der Verhaltenstherapie in Arbeitsgruppen ausgelagert. Hinzu kommt, daß nur wenige Konzepte empirisch validiert wurden. Auch wurde die Frage, wie psychotherapeutische Techniken für die jeweiligen Geschlechter aufgrund dieser Erkenntnisse zu modifizieren sind, bisher kaum untersucht (vergleiche dazu Riecher-Rössler, 2003, Belz und Riecher-Rössler 2008).

\section{Interpersonelle Beziehungen und Kontextbedingungen}

Neben den gesellschaftlichen und kulturellen Rahmenbedingungen, in denen Patienten und Patientinnen sozialisiert wurden und werden, spielen auch sehr individuelle Kontextbedingungen auf der Ebene ihrer aktuellen interpersonellen Beziehungen eine Rolle. So ist es wichtig zu wissen, wie weibliche und männliche Bezugspersonen des Patienten bzw. der Patientin mit der psychischen Störung umgehen. Welche gesellschaftlich geltenden Normen und Bewertungen werden in der konkreten Lebenswelt der Patienten und Patientinnen geteilt? Werden spezifische Verhaltensweisen, die für die Aufrechterhaltung einer Störung mitverantwortlich sind, vom Ehepartner als positiv und 
richtig bewertet, wie z.B. Hilflosigkeit und Abhängigkeit von Frauen bei Angststörungen und Depressionen? Werden Verhaltensweisen in unterschiedlichen Lebensbereichen auch unterschiedlich bewertet und entsprechend konflikthaft erlebt (z.B. assertives Verhalten einer Frau, das am Arbeitsplatz passend ist, wird in der Familie als unweiblich, aggressiv und herzlos kritisiert)? Unterliegen spezifische Verhaltensweisen, die in einem anderen kulturellen Kontext selbstverständlich zur männlichen oder weiblichen Rolle gehören und dort Beziehungen prägen, in einer neuen Umgebung mit neuen Beziehungen auch anderen Bewertungsmustern (z.B. Migranten und Migrantinnen, binationale Ehen)? Wie konstituiert sich also Gesellschaftliches und Kulturelles im Privaten, und wie beeinflußt dieser individuelle Kontext die Störung und das psychotherapeutische Geschehen? Wieviel Toleranz existiert in den interpersonellen Beziehungen bezüglich Abweichung und möglicher Veränderung und einem eventuellen Ausbrechen aus den Bewertungsmustern einer Familie oder Gemeinschaft? Daß es dabei geschlechtsspezifische Unterschiede gibt, zeigen die Ergebnisse des Gender-Datenreports von 2005 (Cornelißen 2005). Danach wird Frauen bei abweichendem Verhalten schneller und häufiger das Aufsuchen psychosozialer Dienste nahegelegt bzw. werden durch Dritte korrigierende Interventionen eingeleitet.

Nicht zuletzt sollten geschlechtsspezifische Bedürfnisse im Setting berücksichtigt werden. Oft "gestatten" sich Patientinnen auch nur dann eine Psychotherapie, wenn diese während der Schulzeiten der Kinder angeboten wird oder wenn Betreuungsangebote für Kinder vermittelt werden. Der Partner sollte im Setting nie vergessen werden. Manche Therapien sind zum Scheitern verurteilt, wenn der Partner nicht rechtzeitig einbezogen und für die Therapie gewonnen wird. Auch sollte der Partner - gerade bei Frauen mit Kindern - oft viel stärker als Ressource genutzt werden (vergleiche dazu Belz und Riecher-Rössler, 2008).

Mit der wachsenden Zahl von Flüchtlingen und Migranten und Migrantinnen in Europa nimmt auch die Vielfalt an Lebenskontexten weiter zu, mit denen Psychotherapeuten und therapeutinnen konfrontiert sein werden. Angesichts der gesellschaftlichen, kulturellen und religiösen Vielfalt der verschiedenen Herkunftsländer und Regionen mit ihren jeweils eigenen geschlechtsspezifischen Rollenvorstellungen, aus denen die Patienten und Patientinnen kommen, dürfte es für Therapierende noch notwendiger sein, die eigenen Geschlechtsrollenstereotypien und geschlechtsspezifischen Prägungen fortlaufend zu reflektieren.

\section{Therapiebeziehung}

In der therapeutischen Beziehung sind sowohl das biologische als auch das soziale Geschlecht des Therapeuten und der Therapeutin und die damit zusammenhängenden geschlechtsspezifischen Erwartungen an Patientinnen und Patienten hinsichtlich Partnerschaft, Arbeitsfähigkeit, Familienleben, Sexualität etc. von Belang.

\section{Studien zum Geschlecht der Therapierenden und zur geschlechtsspezifischen Passung}

Rudolf (2002) untersuchte den Einfluß des Geschlechts von Therapeuten und Therapeutinnen auf die Therapie. Es wurden stationär behandelte Patienten und Patientinnen mit sozialer Ängstlichkeit untersucht, bei denen gleichzeitig Beziehungsängste sowie eine Verunsicherung der Geschlechtsidentität vermutet wurden. Hier zeigte sich ein deutlich besseres Ergebnis bei den männlichen Patienten, die von weiblichen Therapeutinnen behandelt wurden, als bei allen anderen Geschlechterkombinationen zumindest gemessen an der Symptomverringerung auf der Skala „Ängstlichkeit im Kontakt“. Im Gegensatz dazu wurde aber aus Perspektive von Patienten und Patientinnen gegen Ende der Therapie die Arbeitsbeziehung am besten in der Konstellation weibliche Patientin/männlicher Therapeut eingeschätzt.

Bowman et al. (2001) haben mit Hilfe einer Metaanalyse von 58 Studien den Effekt des Geschlechts von Therapeuten und Therapeutinnen auf das Behandlungsergebnis 
berechnet. Die Autoren fanden eine zwar signifikante, aber nur geringe Überlegenheit für Therapeutinnen $(d=0,04)$, wobei das Geschlecht der Patienten keine Rolle spielte. Auch Beutler et al. (2004) fanden in einem Überblick über zehn Studien, daß die Bedeutung des Geschlechts des Therapierenden oder die Zuordnung zwischen Patient/Patientin und Therapierenden nach Geschlecht für das Therapieergebnis (einschließlich Dropout) geringer sei, als bislang berichtet.

In der Studie von Owen et al. (2009) erzielten die einen Therapierenden durchgehend bessere Ergebnisse mit Männern, andere hingegen mit Frauen. Es scheint also bei Therapierenden eine spezifische Genderkompetenz bzw. -präferenz zu geben, die das Therapieergebnis beeinflußt.

Fragt man Patienten und Patientinnen nach dem von ihnen präferierten Geschlecht des Therapeuten, so ist das Geschlecht des Therapierenden dem überwiegenden Teil egal. Es gibt allerdings naheliegende themenspezifische Ausnahmen wie sexueller Mißbrauch, Gewalterfahrung oder eine konfliktbeladene Beziehung zu einem Elternteil. Hier sollten Patienten und Patientinnen das Geschlecht des Therapierenden frei wählen können (Leitner 2014).

\section{Studien zu Geschlechtsrollenstereotypen von Therapierenden}

Auch Therapierende haben herkömmliche und alltäglich eingeübte Bilder von Männlichkeit und Weiblichkeit. Welche Bedeutung Geschlechtsrollenstereotypen auf Seiten der Therapierenden für das Therapieergebnis haben können, zeigt sich in folgenden Studien.

Rudolf (2002) legte hierzu interessante Ergebnisse vor. Seine Studien basieren auf fast 2000 ambulanten und stationären Patientinnen und Patienten in psychoanalytischer Therapie. Dabei zeigte sich, daß Therapeutinnen generell weniger als Therapeuten dazu neigten, Auffälligkeiten bei Patientinnen und Patienten als pathologisch zu gewichten. Die ausgeprägtesten psychopathologischen Befunde wurden von männlichen Therapeuten bei Patientinnen gesehen. Therapeuten stuften Frauen also als besonders krank ein. Umgekehrt sahen Therapeutinnen bei männlichen Patienten die geringste Symptomausprägung. Sie erlebten Männer also als vergleichsweise weniger krank. Therapeutinnen nannten bei ihren Patientinnen auch häufiger positive Merkmale wie „,spontan“, „,wandlungsfähig“, „,beweglich“, ,erfinderisch“, „,mitfühlend“, ,"gefühlvoll“, während männliche Therapeuten bei ihren Patientinnen häufiger die negativen Gegenpole ankreuzten (naiv, schwerfällig, matt, einfallslos usw.). Männliche Therapeuten schätzten weibliche Patientinnen, vor allem aber auch männliche Patienten, sehr viel häufiger als narzisstisch-kämpferisch ein, als Therapeutinnen dies taten.

Therapeuten und Therapeutinnen therapieren umso erfolgreicher und ihre Patienten und Patientinnen sind umso zufriedener, je weniger konservativ die von ihnen vermittelte Einstellung zu Geschlechterrollen ist (Tokar et al. 2000). Auch die Geschlechtsrollenstereotypen von Patienten und Patientinnen können für die therapeutische Arbeit relevant sein. Dies zeigen Studien, die an traditionell orientierten Männern in amerikanischen Militärkrankenhäusern für Kriegsveteranen gemacht wurden (Deering und Gannon 2005). Das Geschlecht des Therapierenden entscheidet bei traditionell orientierten Männer mit darüber, ob sie überhaupt bereit sind, ihre häufig skeptische Haltung gegenüber Psychotherapie aufzugeben und sich auf die Patientenrolle einzulassen. Bei Männern, die wegen häuslicher Gewalt an Therapiegruppen teilnahmen (Päivinen 2010), kann eine Therapeutin entweder den Teilnehmern helfen, Empathie gegenüber den Frauen, die Gewaltopfer geworden sind, zu entwickeln oder aber auch genau dies verhindern, wenn Männer Mühe haben, die Therapeutin in ihrer professionellen Rolle zu akzeptieren und sie "nur" als Frau sehen. Dies zeigt, wie komplex das Zusammenspiel zwischen Therapierenden und Patienten sowie Patientinnen im Therapieprozeß sein kann und daß allein das Geschlecht und die Störung noch nicht 
darüber entscheiden, welche Kombination am besten ist. Deering und Gannon weisen (2005) darauf hin, daß Therapierende, die mit einer Welt vertraut sind, in der Geschlechtsrollen zunehmend androgyner werden, es möglicherweise unterschätzen, wie fremd für "traditionelle“ Männer Psychotherapie sein kann. Das Wissen um diese spezifischen Herausforderungen sollte in den Umgang mit Widerstand, Übertragung und Gegenübertragung einbezogen werden.

Die referierten Ergebnisse zeigen, daß es vor allem die Kombination von biologischem Geschlecht mit den vom Therapierenden genauso wie von Patient und Patientin transportierten Geschlechtsrollenstereotypen ist, die die Therapiebeziehung, die therapeutischen Möglichkeiten und damit letztendlich den Therapieerfolg mitbestimmt. Diese Zusammenhänge sind gerade für weitere Forschungsbemühungen hochrelevant. Zukünftig sollten genau diese komplexen Wechselwirkungen zwischen verschiedenen geschlechtsspezifischen Merkmalen von Patienten und Patientinnen und einem sich spezifisch darauf einstellenden, d.h. responsiven Therapierenden untersucht und entsprechend differentiell ausgewertet werden (Caspar und grosse Holtforth 2009). Erst dann kann beantwortete werden, bei welchen Kombinationen ein Berücksichtigen geschlechtsspezifischer Variablen notwendig und erfolgversprechend ist und was es auf Seiten des Therapierenden braucht, um dies auch anwenden zu können. Ein interessanter schulenübergreifender Ansatz, der zeigt wie Responiveness individuell umgesetzt werden kann und mit dem es möglich ist, auch die Funktionalität geschlechtsrollen(non)konformen Verhaltens (z.B. betont "männlich" auftreten, um nicht als Schwächling dazustehen etc.) zu verstehen ist die motivorientierte Beziehungsgestaltung (Caspar, 2008). Sie erlaubt Therapierenden, sich auf die Motive einzustellen, die den geschlechtsrollenstereotypen Verhaltensweisen zugrunde liegen (z.B. sichere dir die Anerkennung deiner Peers).

\section{Mißbrauch in der Psychotherapie}

In einer neueren Studie zu den Risiken und Nebenwirkungen von Psychotherapie antworten die befragten Patientinnen, daß sie die Dyade von weiblicher Patientin und männlichem Therapeuten als mit mehr Risiken verbunden ansehen als andere Kombinationen von Therapie (Leitner et al. 2014).Dies dürfte u.a. damit zu tun haben, daß sexuelle Übergriffe in der Psychotherapie v.a. zwischen männlichen Therapeuten und Patientinnen vorkommen. Bezüglich dieses überaus wichtigen geschlechtsspezifischen Aspekts der Psychotherapie sei auf einschlägige Literatur verwiesen (DGVT 2013, Franke \& Riecher-Rössler 2013).

\section{Therapietechniken und -ziele}

\section{Geschlechtsspezifische Wirksamkeit herkömmlicher Psychotherapien}

Die klassischen, also nicht geschlechtsspezifisch modifizierten Psychotherapieverfahren und -techniken wurden bezüglich ihrer geschlechtsspezifischen Wirksamkeit bisher kaum verglichen (Übersicht in Riecher-Rössler 2003, Belz und Riecher-Rössler 2008).

In den wenigen diesbezüglichen Studien zur kognitiven Verhaltenstherapie (KVT) fanden sich bis auf eine Ausnahme keine Geschlechtsunterschiede in der Wirksamkeit. In dieser einen Studie war bei schwerer Depression die kognitive Verhaltenstherapie bei Frauen weniger wirksam als bei Männern. Besonders kognitiv-verhaltenstherapeutische Gruppentherapie scheint bei Frauen gut akzeptiert zu sein.

Auch für die interpersonelle Psychotherapie (IPT) gibt es keinen klaren Nachweis einer geschlechtsunterschiedlichen Wirksamkeit, obwohl sie bei Frauen besonders häufig eingesetzt wird. Es wurde spekuliert, dass die IPT von Frauen besser akzeptiert als die KVT, da in der IPT neben Interpersonellem auf Emotionales und Rollenaspekte sowie Rollenübergänge fokussiert wird, während es bei der KVT eher um Strukturelles und Rationales geht. Inwieweit in diese Überlegungen ebenfalls Geschlechtsrollenstereotype einfliessen, ist kritisch zu diskutieren. 
Leff et al. (2000) fanden, daß auch Paartherapie bei Frauen mit Depression wirkt. Die Studie wurde allerdings nur bei Frauen durchgeführt, deren Partner zuvor als „kritisch“ eingestuft worden waren. Sie zeigt, daß es unter Umständen hilfreich sein kann, die Paarbeziehung in der differenziellen Indikationsstellung bezüglich der Art der Psychotherapie zu berücksichtigen.

\section{Störungenspezifische Psychotherapien für Frauen}

Zwar haben es die einzelnen Psychotherapierichtungen bisher eher vernachlässigt, allgemeingültige geschlechtsspezifische Modifikationen ihrer psychotherapeutischen Techniken zu entwickeln, doch gibt es für einzelne Störungsbilder inzwischen spezifische Therapieformen für Frauen (Übersicht bei Riecher-Rössler 2003, Belz und Riecher-Rössler 2008). Dabei handelt es sich meist um Therapien, die nicht nur störungs-, sondern auch themenspezifisch sind, die also nicht nur auf eine spezifische Diagnose, sondern oft auch auf eine spezifische Problematik abgestimmt sind, etwa Probleme der Peripartalzeit, des Prämenstruums oder auf einen Zustand nach Vergewaltigung.

So wurde etwa für die Psychotherapie von Schwangeren die IPT, die klassisch vor allem die vier Themenbereiche „Trauer", ,,interpersonelle Konflikte“, „, Rollenübergänge“ und „interpersonelle Defizite“ kennt, um den Problembereich „Schwangerschaft" ergänzt und evaluiert.

Auch für die postpartale Depression wurden inzwischen mehrere spezifische Konzepte entwickelt, für die zum Teil auch schon ein Wirksamkeitsnachweis erbracht wurde. Unter anderem wurde eine spezifische, manualisierte Form der IPT für Mütter mit Depression vorgelegt. Auch verschiedene Gruppentherapien wurden entwickelt, so eine spezifische kognitive Gruppentherapie oder eine spezifische Gruppentherapie, die Elemente aus verschiedenen Psychotherapieformen enthält und gleichzeitig auch die jeweiligen Kinder und Väter einbezieht (Übersicht in Riecher-Rössler 2003, Belz und Riecher-Rössler 2008). Riecher-Rössler hat an ihrer Klinik eine spezifische Gruppentherapie für Depression in der frühen Mutterschaft entwickelt: eine Gruppentherapie mit überwiegend kognitivverhaltenstherapeutischen, aber auch psychoedukativen und systemischen Elementen, die in manualisierter Form vorliegt (Hofecker Fallahpour et al. 2005). Auch hier zeigte sich eine gute Wirksamkeit. Appleby (2001) konnte die Effektivität einer von ihm entwickelten „kognitiv-behavioralen Beratung" nachweisen.

Für das prämenstruelle Syndrom wurde eine themenzentrierte kognitive Verhaltenstherapie entwickelt und gezeigt, daß diese wirksamer ist als reine Beratung. Auch eine Bewältigungstrainingsgruppe und Entspannungstraining haben sich hier als hilfreich erwiesen (Übersicht in Riecher-Rössler 2003, Belz und Riecher-Rössler 2008).

Die meisten der bisher vorgelegten Studien zu spezifischen Psychotherapieformen für Frauen zeigen jedoch erhebliche methodische Probleme und unterschieden häufig nicht zwischen dem biologischen und dem psychosozialen Geschlecht bzw. setzten beides gleich. Meist wurden nur sehr kleine Patientenzahlen behandelt, die Ein- und Ausschlußkriterien wurden häufig nicht klar genannt, Diagnosen wurden zum Teil nicht standardisiert erhoben. Kontrollgruppen fehlten entweder ganz, oder die Zuteilung zur Therapie- versus Kontrollgruppe erfolgte nicht randomisiert. Die Beurteiler des Therapieerfolgs waren meist nicht verblindet. Auch wurden oft keine Interraterreliabilitätsstudien referiert. Vor allem aber wurde der Einfluß zusätzlicher Medikation oft nicht kontrolliert, die Verweigerer- und Dropout-Raten werden nicht angegeben oder nicht kritisch diskutiert, die Interventionen oft nur unzureichend beschrieben und die Studien häufig nur in schlecht zugänglichen Organen publiziert (Übersicht bei Riecher-Rössler 2003, Belz und Riecher-Rössler 2008).

\section{Allgemeine Prinzipien einer geschlechtersensiblen Psychotherapie}


Im Diskurs der feministischen Psychotherapie, die weniger eine Psychotherapierichtung als eine bestimmte Haltung vertritt, werden einige Punkte betont, die generell in der Psychotherapie von Frauen mehr Gewicht haben sollten (Willutzki 2001). So soll die Psychotherapie bei Frauen ihren spezifischen soziokulturellen Kontext berücksichtigen, also etwa die geschlechtsspezifische Sozialisation, die geschlechtsspezifischen Stressoren, den speziellen sozialen Status der Frau usw. Einschätzungen von Frauen sollen nicht von vornherein als verzerrt oder dysfunktional bewertet werden, vielmehr sollen mit den Augen der Frau deren soziale Realität betrachtet und so auch ihre emotionalen Reaktionen verstanden werden.

Ziel ist das „Empowerment" von Frauen. Gefördert werden soll dies durch hohe Transparenz in der Therapie, möglichst wenig hierarchische Beziehungsgestaltung und wenig belehrende Elemente, eine möglichst starke Einbeziehung der Patientin in die Therapieplanung und den therapeutischen Prozeß sowie durch eine allgemeine Förderung von Aktivitäten. Patientinnen sollen in der Therapie auch speziell zur Nutzung ihrer vielen positiven inneren und äußeren Ressourcen angeregt werden (Übersicht bei Riecher-Rössler 2003, 2013). Die Skizzierung einer Psychotherapie für Männer bzw. männerspezifische Interventionsstrategien finden sich in Cochran und Rabinowitz (2000), Neumann und Süfke (2004) sowie Nahon und Lander (2014).

Sowohl für Männer wie für Frauen ist es sinnvoll, geschlechtsstereotypes Rollenverhalten und entsprechende Einstellungen zu hinterfragen und dort zu reduzieren, wo sie dysfunktional und störungsaufrechterhaltend wirken. Zentrale Themen für Frauen in Psychotherapie sind oft die Steigerung von Selbstbewußtsein, Eigenständigkeit, Durchsetzungs- und Abgrenzungsfähigkeit, Beharrlichkeit, Zielstrebigkeit, das Äußern von Kritik und Wünschen, Initiative zeigen, mehr Zusammenhalt und weniger Konkurrenz mit Frauen. Wichtige Therapieziele für Männer sind die Verbesserung der Wahrnehmung und das Ausdrücken von Gefühlen, die Steigerung von emotionalem Austausch, Annahme fremder Hilfe aber auch von Kritik, Änderung des Risikoverhaltens, Entwicklung sozialer Sensibilität und Teamfähigkeit, Übernahme von mehr sozialer Verantwortung, das Äußern von Zweifel und Eingestehen von Fehlern. Die genannten Aspekte sind eine Themensammlung, die als Hintergrundfolie in der therapeutischen Arbeit genutzt werden kann, wohl wissend, daß sich das Bild im Einzelfall ganz anders darstellen kann.

Folgende Vorgehensweisen können dabei von Therapierenden sinnvoll eingesetzt werden:

- Hinterfragen von Spannungen, die durch zugeschriebene und konfligierende Geschlechtsrollen und -identitäten auftreten.

- Einüben von Rollenanteilen, die den eigenen Fähigkeiten, Neigungen und Möglichkeiten entsprechen.

- Wecken von Interesse für die Attraktivität von Rollenvielfalt und flexiblen Bewältigungsansprüchen.

\section{Ebene der Selbsterfahrung}

Wenn traditionelle Geschlechtsrollenstereotypen zu ungünstigen Therapieverläufen beitragen können, ist es unabdingbar, daß Therapierende sich schon während ihrer Ausbildung darüber klar werden, wo sie selbst bezüglich Geschlechterfragen besonders empfindsam oder verführbar sind und was das mit der eigenen geschlechtsspezifischen Sozialisation zu tun hat. Selbsterfahrung sollte also auch die Geschlechtsrollenorientierung und Stereotypen auf ihrem biographischen Hintergrund klären. Besonders wichtig scheint auch, Therapierende - vor allem jüngere Therapeuten - in ihrer Ausbildung spezifisch für "frauentypische" genauso wie "männertypische“ Konflikte und Plausibilitätsfallen zu sensibilisieren, beispielsweise solche, die sich bei Rollenübergängen, etwa dem zur Elternschaft, ergeben. Rochlen (2005) weist auf prinzipielle Inkongruenzen zwischen der 
Therapiekultur und der männlichen Ideologie von Maskulinität hin, welche einen Einfluß darauf haben kann, ob und inwieweit der Beruf des Therapeuten attraktiv ist

Schließlich sollten Konflikte auf dem Hintergrund von Unterschieden im emotionalen Ausdruck zwischen Männern und Frauen verstanden werden. Damit ist gemeint, daß Alltagskonflikte häufig nicht zuletzt auch deshalb entstehen oder sich verschärfen, weil die beteiligten Männer und Frauen sich verschieden ausdrücken bzw. unterschiedlich kommunizieren. Hier gilt es, auch eigene geschlechtsspezifische Kommunikationsmuster kennenzulernen und zu reflektieren

\section{Psychotherapieforschung}

Inzwischen erobert sich das Genderthema vor allem in den Lehrbüchern der klinischen Psychologie, der Gesundheitspsychologie und der Medizin einen festen Platz (Franke und Kämmerer 2001; Riecher-Rössler und Bitzer 2005; Schigel 2012; Christ 2013: Boothe und Riecher-Rössler, 2013; Wimmer-Puchinger et al., 2016). In den letzten Jahren sind zahlreiche Publikationen erschienen, die gesellschaftliche Fragen und spezielle gesellschaftliche Gruppierungen als Thema der Psychotherapie entdeckt haben (z.B. alte Menschen, Frauen, Männer, Flüchtlinge sowie Migranten und Migrantinnen). Diese Interessenverschiebung entspricht einem generellen Wertewandel in unserer Gesellschaft. Bedauerlicherweise nimmt die Psychotherapieforschung hier keine führende Rolle ein, sondern folgt dieser Entwicklung lediglich (Beutler et al. 2004). Nach wie vor fehlen Psychotherapiestudien mit differenzierteren Designs, die der Komplexität der Thematik gerecht werden könnten, auch 2016 weitgehend. Bislang liegt der Schwerpunkt auf Studien, die subjektive Einschätzungen und Präferenzen von Patienten und Patientinnen erfragen, ohne den Therapieoutcome zu erfassen. Nach wie vor werden in Publikationen viele Annahmen zur Bedeutung geschlechtsspezifischer Variablen für den Therapieerfolg getroffen, bis hin zur Empfehlung spezifischer Therapieformen, die um diese Konzepte herum zu entwickeln wären. Diese Annahmen sind jedoch nicht von tatsächlichen Forschungsergebnissen begleitet, die belegen, daß diese Behandlungsformen tatsächlich wirksam sind.

Letztlich geht es um die Frage, ob unsere Therapien besser werden, wenn wir die komplexen geschlechtsspezifischen Besonderheiten, die das Leben von Männern und Frauen prägen, in unsere Fallkonzeptionen einbeziehen. Das, was wir an Daten haben, scheint dies nahezulegen, gleichzeitig wissen wir aber noch zu wenig, um das im Detail empirisch belegen und anwenden zu können.

Eine Zusammenstellung im Kasten 28-1 zeigt (vgl. dazu u.a. Kämmerer 2001, Belz und Riecher-Rössler 2013), welche Probleme und Defizite in bisherigen Studien zu finden und zukünftig zu vermeiden sind, wenn wir der Bedeutung der Variable Geschlecht besser als bisher gerecht werden wollen.

\section{Kasten 28-1.}

\section{Geschlechtsspezifische Erhebungs- und Interpretationsfehler}

- Geschlechterinsensibilität Geschlechterdifferenz wird ignoriert; Themen werden generalistisch behandelt; ätiologische Modelle basieren auf Befunden, die nur an einem Geschlecht erhoben worden sind; keine differenzielle Auswertung von psychotherapeutischen Interventionen.

- Familialismus Unterform der Geschlechterinsensibilität; Familie als Einheit ohne Berücksichtigung der unterschiedlichen Effekte von Aufgaben auf Einzelne, z.B. Pflege 
von Angehörigen.

- Geschlechterdichotomie Unterschiede werden überbetont, Überschneidungen vernachlässigt; Stereotypien werden als Gesundheitsnorm verwendet, z.B. Maskulinitäts- und Feminitätsskalen in Tests.

- Doppelter Bewertungsmaßstab Anwendung verschiedener Maßstäbe für gleiche Charakteristika: z.B. aggressives Verhalten - bei Jungen sozial angesehen, bei Mädchen problematisch.

- Geschlechterverklärung Überhöhung empirisch gefundener Geschlechtsmerkmale zur Legitimierung normativer Forderungen: emotionale Expressivität von Frauen als Maßstab für emotionale Ausdrucksfähigkeit von Männern.

- Gleichsetzung Sex und Gender Unterscheidung von biologischem und sozialem Geschlecht findet nicht statt. Design und/oder Auswertung berücksichtigen z.B. nur das biologische Geschlecht und präsentieren die Ergebnisse als genderspezifisch.

- Reduktionistisches Verständnis von Geschlecht Das Geschlecht wird rein deskriptiv und als bloße biologische/demographische Kategorie untersucht, soziale, kulturelle und psychologische Begleiterscheinungen des Geschlechts werden vernachlässigt.

- Varianzvernichtung durch ungeeignete Auswertung Auswirkungen von therapeutischer Responsiveness, d.h. eines sich Einstellens auf den Einzelfall und dessen geschlechtsbezogene Besonderheiten werden wegmittelt

\section{Aus- und Weiterbildung}

Derzeit sind in Deutschland etwa zwei Drittel der psychologischen Psychotherapeuten weiblich und ungefähr $80 \%$ der Psychologiestudierenden. Über die Gründe für diese zunehmende "Feminisierung" des Berufsfelds Psychotherapie ist viel spekuliert worden, empirische Daten sind jedoch Mangelware (Jaggi 2014). In Deutschland, wo Psychologie dem Numerus clausus unterliegt, sind dies womöglich auch Folgen der besseren Schulabschlüsse von Mädchen. Aber auch in Ländern, die keine Aufnahmebeschränkungen fürs Psychologiestudium kennen, studieren zunehmend mehr Frauen Psychologie. Evtl. spielen aber auch geschlechtstypische Gründe für diese Berufswahl eine Rolle, z.B. da Männer Studium und Beruf eher karriere- und statusorientiert wählen, während Frauen in erster Linie Beruf und Familie vereinbaren wollen.

In den leitenden Funktionen im Gesundheits- und Hochschulbereich sind Psychologinnen im Vergleich zu ihren männlichen Kollegen nach wie vor deutlich unterrepräsentiert. Dazu passend werden an Psychotherapieausbildungsinstituten auch überwiegend Frauen von Männern ausgebildet, die mehrheitlich die Ausbildungsinstitute leiten und deren Gremien besetzen und somit im Wesentlichen bestimmen, welche Themen und Perspektiven in den Psychotherapie-Ausbildungscurricula dominieren. Obwohl es also zu einer zunehmenden Feminisierung des Berufs kommt, kann nicht davon ausgegangen werden, daß sich dies auch in einer veränderten Berücksichtigung der Geschlechterperspektive in den Ausbildungsinhalten widerspiegelt.

Folgende Themen sollten Teil der Psychotherapieausbildung sein:

- Verwendung einer gendersensitiven Sprache.

- Integration geschlechtsrelevanter Themen ins reguläre Curriculum: geschlechtsspezifische Sozialisation und Unterschiede in der Entwicklungspsychopathologie, Geschlechtsrollenstereotype, Geschlechtsunterschiede bei psychischen Störungen inkl. Ätiologie, Aufrechterhaltung und Behandlung, sexuelle Belästigung und geschlechterbasierte Gewalt, geschlechtertypische 
Konfliktsituationen, Rollenübergänge etc., Geschlechtsunterschiede im Ausdruck von Emotionen und in der Kommunikation mit möglichen

"Geschlechtermissverständnissen", allgemeine Prinzipien einer geschlechtersensiblen Psychotherapie

- Selbsterfahrung: Sensibilisierung für die eigene geschlechtstypische Sozialisation, für eigene Geschlechtsrollenstereotype etc.

- Supervision: verstärkte Berücksichtigung geschlechtsrelevanter Aspekte in der Problematik der Patientinnen und Patienten, aber auch in der therapeutischen Beziehung inkl. Umgang der Therapierenden mit Macht und Abhängigkeit, Erotik und Sexualität sowie Risiken des Übergriffs und Mißbrauchs innerhalb der Therapie oder in anderen Abhängigkeitsverhältnissen

- Prägnantere Vertretung von Frauen in der Lehre und Leitung von Ausbildungsinstituten und Erarbeitung von Curricula.

\section{Grenzen und Risiken der geschlechtersensiblen Psychotherapie}

Die verschiedenen referierten Theorien, Befunde und eine darauf aufbauende geschlechtersensible Psychotherapie haben auch ihre Grenzen und Risiken (vergleiche dazu Riecher-Rössler 2005, Belz und Riecher-Rössler 2008, 2013). In der Forschung zu geschlechtsspezifischen Einflußfaktoren gibt es bisher fast nur Befunde bezüglich der Depression. Vielen Studien mangelt es noch an methodischer Exaktheit, und Interpretationen sind nicht immer eindeutig möglich. So ist z.B. bezüglich der angeblich negativeren Kognitionen bei Frauen noch nicht sicher, inwieweit die Forschung ein Geschlechtsrollenstereotyp unkritisch perpetuiert: Zum Teil wurde nicht kontrolliert, inwieweit die Probandinnen einfach im Sinne der sozialen Erwünschtheit geantwortet haben, also z.B. ihre Schuldgefühle eher erwähnt und stärker betont haben, schlicht weil dies von Frauen so erwartet wird.

Auch die Kausalkette ist oft nicht ganz klar. So wird etwa ein vergleichsweise passiver Bewältigungsstil schon bei kleinen Mädchen beobachtet, die Depressionshäufigkeit steigt aber erst nach der Pubertät. Hier scheinen also weitere Einflußfaktoren eine Rolle zu spielen. Evtl. ist es aber auch einfach nur so, daß es dauert, bis ungünstige Bewältigungsstile Depression entstehen lassen. Das heißt, die Theoriebildung berücksichtigt zu wenig die biopsychosozialen Interaktionen, ebenso wie sie oft auch die soziale Realität vernachlässigt.

Die Gefahren einer unkritischen Theoriebildung sind vielfältig (vergleiche dazu RiecherRössler 2005, Belz und Riecher-Rössler 2013). Wenn zum Beispiel die geschlechtsspezifische Sozialisation und reale soziale Benachteiligungen vernachlässigt werden, werden gefundene Geschlechtsunterschiede leicht fehlinterpretiert: Auffälligkeiten werden per se auf das biologische Geschlecht anstatt auf die mit dem Geschlecht assoziierten psychosozialen Einflußfaktoren attribuiert. Im Extremfall können die durch solche Forschung gefundenen "Geschlechtsunterschiede" dann in einer Art Zirkelschluß sogar zur Rechtfertigung sozialer Ungleichheit verwandt werden. Dabei ist zu bedenken, daß das Geschlecht im Sinne eines psychosozialen „Gender" auch "gemacht" wird. Von West und Zimmermann wurde in den 80-er Jahren hierfür der Begriff des „doing-gender“ geprägt (Gildemeister 2004), was in etwa bedeutet, daß Frauen (und Männer) dazu tendieren, sich so zu verhalten, wie sie sich laut Geschlechtsrollenstereotyp verhalten sollen.

Schließlich konstelliert sich in der Forderung nach einer geschlechtersensiblen Therapie die Diskussion um den Nutzen störungsspezifischer Therapieansätze und die Vor- und Nachteile manualisierter Therapieprogramme im Allgemeinen. In den letzten Jahren wurde die zunehmende Aufteilung psychischer Probleme in ständig neue und immer spezifischere 
Störungsbilder mehrfach kritisiert, da sie die Gemeinsamkeiten und Überschneidungen zwischen den Störungsbildern sowie häufig vorhandene Komorbiditäten vernachlässigt (Castonguay und Beutler 2005). Insbesondere Therapiemanuale suggerieren, man könne, abgelöst von gesellschaftlichen und lebensweltlichen Bedingungen, eine für alle Patientinnen und Patienten gleichermaßen wirksame Methode zur Behandlung verschiedener Störungsbilder, unabhängig von Geschlecht, Alter und Ethnizität entwickeln.

Ebenso unangemessen wäre es, davon auszugehen, dass Frauen und Männer für sich genommen homogenen Gruppen angehören, deren Mitglieder untereinander alle einheitlich sind. Vielmehr gibt es große individuelle Unterschiede innerhalb der Geschlechtergruppen, und die gefundenen Unterschiede sind nur Mittelwertsunterschiede. Die Überlappung zwischen Männern und Frauen, das Gleiche, das Ähnliche, sind sicherlich sehr viel stärker als die Unterschiede. Wenn die Unterschiede zu sehr betont und vor allem in ihrer Ursache nicht kritisch hinterfragt werden, so kann dies zur Perpetuierung von Geschlechtsrollenstereotypen führen.

Wie kann dann ein vernünftiger Umgang mit den Variablen Sex und Gender aussehen? Unseres Erachtens braucht es für die Behandlung von Frauen und Männern keine geschlechterspezifischen Ansätze. Einleuchtender erscheint vielmehr ein allgemeiner Ansatz, innerhalb dessen sich eine Reihe von Prinzipien auf Gender-Aspekte beziehen. Die entsprechende therapeutische Haltung, die geschlechtsrollentypische Defizite und Fixierungen, welche zur Entstehung und Aufrechterhaltung einer Störung beitragen, aufdeckt und bearbeitet, sollte in jeder Therapie bei beiden Geschlechtern realisiert sein. Dies geht von einem dimensionalen Konzept aus, wonach bei Männern und Frauen spezifische Ausprägungen von Merkmalen, Haltungen und lebensweltlichem Kontext therapierelevant sein können und das Geschlecht einfach Hinweise gibt, mit welchen Ausprägungen und Faktoren eher zu rechnen ist.

Die Politik hat hier bereits wichtige Schritte eingeleitet, denen die psychotherapeutische Praxis und Forschung dringend folgen sollte. Die EU und viele europäische Länder verfolgen inzwischen das Gender Mainstreaming. Ziel dieser Strategie ist es, alle Aktivitäten auf Politik- und Praxisebene daraufhin zu überprüfen, ob sie den spezifischen Belangen von Frauen und Männern gerecht werden und zum Abbau geschlechtlicher Ungleichheit beitragen. Bezogen auf das Gesundheitssystem bedeutet dies, alle gesundheitsbezogenen Interventionen auch daraufhin zu untersuchen, ob sie den Bedürfnissen von Frauen und Männern gleichermaßen Rechnung tragen und die Unterschiede in der Gesundheitssituation von Frauen und Männern zu verringern vermögen.

\section{Literatur}

Belz M, Riecher-Rössler A (2008). Geschlechtsspezifische Aspekte in der Psychotherapie. In S. Herpertz, F. Caspar \& Mundt, Ch. (Hrsg.), Störungsorientierte Psychotherapie. Elsevier, Urban \& Fischer: München.

Belz M, Riecher-Rössler (2013): Welcher Psychotherapiebedarf für wen geschlechtsspezifische Aspekte. In Boothe B, Riecher-Rössler A (Hrsg.). Frauen in Psychotherapie. Grundlagen - Störungsbilder - Behandlungsangebote. Schattauer: Stuttgart.

Bengel J, Belz-Merk M (1997) Subjektive Gesundheitsvorstellungen. In R Schwarzer (Hrsg.): Gesundheitspsychologie. Ein Lehrbuch. Göttingen: Hogrefe 23-41.

Beutler L, Malik M, Alimohamed S, Harwood TM, Talebi H, Noble S, Wong E (2004):

Therapist Variables. In M Lambert (ed.), Bergin and Garfield's Handbook of

Psychotherapy and Behavior Change. Wiley: New York, 227-306.

Boothe B, Riecher-Rössler A (Hrsg.) (2013): Frauen in Psychotherapie. Schattauer: Stuttgart. 
Bowman DG, Scogin F, Floyd M, McKendree-Smith N (2001): Effect of therapist sex on outcome of psychotherapy: A meta-analysis. Psychotherapy 38: 142-148.

Caspar F (2008): Motivorientierte Beziehungsgestaltung - Konzept, Voraussetzungen bei den Patienten und Auswirkungen auf Prozess und Ergebnis. In M Hermer , B Röhrle (Hrsg.), Handbuch der therapeutischen Beziehung, Bd 1. Tübingen: dgvt-Verlag.577 558.

Caspar F, grosse Holtforth M (2009): Responsiveness - eine entscheidende Prozessvariable in der Psychotherapie. Zeitschrift für Klinische Psychologie und Psychotherapie, 38 (1), 61-69

Castonguay L, Beutler L (eds.) (2005): Principles of therapeutic change that work. New York: Oxford University Press.

Christ C (2013): Männerwelten : Männer in Psychotherapie und Beratung. Stuttgart : Schattauer, Stuttgart.

Cochran SV, Rabinowitz FE (2000): Men and depression. Clinical and empirical perspectives. Washington: Academic Press

Cornelißen W (Hrsg.) (2005): Gender-Datenreport. 1. Datenreport zur Gleichstellung von Frauen und Männern in der Bundesrepublik Deutschland. Bundesministerium für Familie, Senioren, Frauen und Jugend: Berlin.

Cornelißen W, Dressel C, Lohel V (2005): Erwerbseinkommen von Frauen und Männern. In W Cornelißen (Hrsg.) (2005), 159-223.

Deering CG, Gannon EJ (2005): Gender and psychotherapy with traditional men. American Journal of Psychotherapy, 59 (4), 351-360.

DGVT (2013): Themenheft Grenzverletzungen und Schäden in Psychotherapie und psychosozialer Beratung. Verhaltenstherapie \& Psychosoziale Praxis, 45. Jg., Nr. 4. Tübingen: dgvt-Verlag.

Dorrmann W, Elsner M, Kirin C, Matthey A, Meyer R, Rose A, Scheler S, Scott S, Stieglitz N, Zeh S (2014): Der jährlich stattfindende Boys' Day - Eine Möglichkeit, den Beruf des Psychotherapeuten mehr in die Öffentlichkeit zu bringen. Psychotherapeutenjournal 13 (4), 384-388.

Franke A, Kämmerer A (Hrsg.) (2001): Klinische Psychologie der Frau - Ein Lehrbuch. Göttingen: Hogrefe.

Franke I, Riecher-Rössler A (2013) Missbrauch in der Psychotherapie. In: Boothe B, RiecherRössler A (Hrsg.): Frauen in Psychotherapie. Stuttgart, Schattauer, pp 433-445.

Garcia-Moreno C, Riecher-Rössler A (Eds.): Violence against women and mental health. Basel, Karger, 2013

Gildemeister R (2004): Doing Gender. Soziale Praktiken der Geschlechterunterscheidung. In: R Becker, B Kortendiek (Hrsg.): Handbuch Frauen- und Geschlechterforschung. Theorie, Methoden, Empirie (Geschlecht und Gesellschaft; Bd. 35). Wiesbaden: Verlag für Sozialwissenschaften.

Gutiérrez-Lobos K, Wölfl G, Scherer M, Anderer P, Schmidl-Mohl B (2000): The gender gap in depression reconsidered: the influence of marital and employment status on the female/male ratio of treated incidence rates. Social Psychiatry and Psychiatric Epidemiology 35(5): 202-210.

Hinz A (2007): Prävention durch Unterstützung der Bewältigung geschlechtsspezifischer Entwicklungsaufgaben. Zeitschrift für Pädagogische Psychologie 21 (2), 145-155.

Hofecker Fallahpour M, Zinkernagel C, Frisch U, Riecher-Rössler A (2005): Was Mütter depressiv macht ... und wodurch sie wieder Zuversicht gewinnen. Ein Therapiehandbuch. Bern: Huber.

Holzbach R, Martens M, Kalke J, Raschke P (2010): Zusammenhang zwischen Verschreibungsverhalten der Ärzte und Medikamentenabhängigkeit ihrer Patienten. Bundesgesundheitsblatt 2010 · 53:319-325 DOI 10.1007/s00103-010-1029-8. Online publiziert: 4. März 2010 
IGES Institut (2015): DAK Gesundheitsreport 2015. Hamburg: DAK Versorgungsmanagement Jaggi E (2014): „Weil Frauen ja so emotional sind ...": die Feminisierung eines Berufes Psychotherapeutin. Psychotherapie-Wissenschaft, Jg. 4, Heft 2.

Kämmerer A (2001): Weibliches Geschlecht und psychische Störungen - Epidemiologische, diagnostische und ätiologische Überlegungen. In A Franke, A Kämmerer (Hrsg.) (2001), 51-88.

Khelaifat N, Schröttle M. (2008): Gesundheit - Gewalt - Migration: Eine vergleichende Sekundäranalyse zur gesundheitlichen Gewaltsituation von Frauen mit und ohne Migrationshintergrund in Deutschland. Enddokumentation Juli 2007.

Kofahl C, Mestheneos E, Triantafillou (2005): Zusammenfassende Übersicht der Ergebnisse aus der EUOFAMCARE-Sechs-Länder-Studie. http://www.uke.de/extern/eurofamcare/documents/deliverables/summary of findings de.pdf

Kolip P, Hurrelmann K (Hrsg.) (2016): Handbuch Geschlecht und Gesundheit. Männer und Frauen im Vergleich. Göttingen: Hogrefe.

Langehennig M, Betz D, Dosch E (2012): Männer in der Angehörigenpflege. Weinheim, Basel: Beltz Juventa.

Leff J, Vearnals S, Brewin CR, Wolff G, Alexander B, Asen E, Dayson D, Jones E, Chisholm D, Everitt B (2000): The London Depression Intervention Trial. Randomised controlled trial of antidepressants v. couple therapy in the treatment and maintenance of people with depression living with a partner: clinical outcome and costs. British Journal of Psychiatry 177: 95-100.

Leitner A, Schigl B, Märtens M (Hrsg.) (2014): Wirkung, Risiken und Nebenwirkungen von Psychotherapie. Wien: Facultas.

Mahalik JR, Good GE, Englar-Carlson M (2003): Psychotherapist Gender Competence. Masculinity scripts, presenting concerns and help-seeking: Implications for practice and training. Professional Psychology: Theory, Research and Practice, 34, 123-131.

Meshkat D, Kutzelnigg A, Kasper S Winkler D (2010): Ärgerattacken bei Depressionen: Geschlechtsspezifische Aspekte. Journal für Neurologie Neurochirurgie und Psychiatrie; $11(3), 22-25$.

Möller-Leimkühler AM (2000): Männer und Depression: Geschlechtsspezifisches Hilfesuchverhalten. Fortschritte der Neurologie und Psychiatrie 68: 489-495.

Nahon D, Lander NR (2014): Working with Men in Individual Psychotherapy form an Integrity Model Perspective: The Unsung Heroes. The Journal of Men's Studies, Vol.22, No. 3, $194-206$.

Nesbitt MN, Penn NE (2000): Gender stereotypes after thirty years: a replication of Rosenkrantz et al. (1968). Psychological Report 87: 493-511.

Neumann W, Süfke B (2004): Den Mann zur Sprache bringen. Psychotherapie mit Männern. Tübingen: dgvt.

Owen J, Wong YJ, Rodolfa E (2009): Empirical search for psychotherapists' gender competence in psychotherapy. Psychotherapy, Theory, Research, Practice, Training, 46, $4,448-458$.

Päivinen H (2010): Positions constructed for a female therapist in male batterers' treatment groups. Master's thesis in psychology, Department of Psychology: University of Jyväskylä.

Riecher-Rössler A (2003): Psychotherapie von Frauen - Chancen und Grenzen der Geschlechtersensibilität. Psychodynamische Psychotherapie 2: 91-101

Riecher-Rössler A, Bitzer J (Hrsg.) (2005a): Frauengesundheit. Ein Leitfaden für die ärztliche und psychotherapeutische Praxis. München, Jena, Elsevier, Urban \& Fischer.RiecherRössler A (2005b): Psychotherapie für Frauen. In: Riecher-Rössler A, Bitzer J (Hrsg.) Frauengesundheit. Ein Leitfaden für die ärztliche und psychotherapeutische Praxis. Urban \& Fischer: München, S. 105-115. 
Riecher-Rössler A (2013) Gender-Aspekte. In: Rössler W, Kawohl W (Hrsg.) Soziale Psychiatrie: das Handbuch für die psychosoziale Praxis. Stuttgart, Kohlhammer Verlag, S. 127-141

Riecher-Rössler A (2016): Weibliche Rollen und psychische Gesundheit. In: WimmerPuchinger B, Gutiérrez-Lobos K, Riecher-Rössler A (Hrsg.): Irrsinnig weiblich - Psychische Krisen im Frauenleben. Hilfestellung für die Praxis. Berlin, Heidelberg: Springer-Verlag. RKI - Robert Koch-Institut (2014): Gesundheitliche Lage der Männer in Deutschland. Berlin (Beiträge zur Gesundheitsberichterstattung des Bundes), 2014.

Rochlen AB (2005): Men in (and out) of therapy: Central concepts, emerging directions, and remaining challenges. Journal of Clinical Psychology, 61, 627-631.

Rudolf G (2002): Gibt es nachweisbar Einflüsse der Geschlechtszugehörigkeit in der Psychotherapie? In Schweizer Charta für Psychotherapie (Hrsg.), Mann oder Frau? Tübingen: Edition Diskord, 75-95.

Schigl B (2012): Psychotherapie und Gender - Konzepte, Forschung, Praxis : welche Rolle spielt die Geschlechtszugehörigkeit im therapeutischen Prozess? Integrative Modelle in Psychotherapie, Supervision und Beratung. Wiesbaden : Springer VS, Wiesbaden.

Schwind H-D, Felchenhauer D, Ahlborn W, Weiß R (2001): Kriminalitätsphänomene im Langzeitvergleich am Beispiel einer deutschen Großstadt. Bochum 1975 - 1986 - 1998. Neuwied: Luchterhand.

Seedat S, Scott KM, Angermeyer MC, Berglund P, Bromet EJ, Brugha TS, Demyttenaere K, de Girolamo G, Haro JM, Jin R, Karam EG, Kovess-Masfety V, Levinson D, Medina Mora ME, Ono Y, Ormel J, Pennell BE, Posada-Villa J, Sampson NA, Williams D, Kessler RC (2009): Cross-national associations between gender and mental disorders in the World Health Organization World Mental Health Surveys. Archives of General Psychiatry 66(7): 785795. doi: 66/7/785 (pii); 10.1001/archgenpsychiatry.2009.36

Stürzer M, Cornelißen W (2005): Gesundheitsstatus und Gesundheitsrisiken von Frauen und Männern. In W Cornelißen (Hrsg.) (2005), 470-523.

[WHO] World Health Organisation (2000): Women's mental health: an evidence based review. Genf: World Health Organisation.

Wittchen, H.-U., Jacobi, F., Mack, S., Gerschler, A., Scholl, L., Gaebel, W., Zielasek, W., Maier, W., Wagner, M., Hapke, U., Siegert, J., Höfler, M. (2012): Was sind die häufigsten psychischen Störungen in Deutschland? Erste Ergebnisse der "Zusatzuntersuchung psychische Gesundheit" (DEGS-MHS). Bundesgesundheitsblatt, 9-10.

Willutzki U (2001): Feministische Perspektiven für die psychosoziale Versorgung. In A Franke, A Kämmerer (Hrsg.) (2001), 689-715.

Wolfersdorf M, Schulte-Wefers H (2005): Suizidalität und geschlechtsspezifische Aspekte. In A Rohde, A Marneros (Hrsg.), Geschlechtsspezifische Psychiatrie und Psychotherapie. Ein Handbuch. Stuttgart: Kohlhammer, 485-502. 